\title{
Genital Human Papilloma Virus Infections
}

Rashmi Prasad Yadav

Nepal Academy of Medical Science

\begin{abstract}
Genital HPV infection is commonest viral sexually transmitted disease (STD), which give rise to genital warts. Some of these viruses cause genital lesions which have potential to progress into pre-invasive and subsequently invasive lesions of lower genital tracts. And so, the public awareness regarding this problem needs to be enhanced; so as to prevent development of malignancy in them and to improve the quality of reproductive health in these women and her partner(s).
\end{abstract}

Keywords: HPV, Genital wart, STD

\section{Introduction}

Genital human papilloma virus (HPV) infections have various presentations in women. They can cause new growths in the form of genital warts or condyloma acuminata on the cornified squamous skin of the perineum, on perianal region or on the mucous membrane of the introitus, the vagina, or the cervix. The appearance of these lesions is cause of concern for the women involved. As these lesions are marker of sexual indiscretion practiced by the patients, they want the lesions to be removed as soon as possible. The women in whom mucous membrane warts are seen, they present with the symptoms of dyspareunia. Besides, present available methods of treatment are not satisfactory. The treatment offered to them are uncomfortable and post treatment scarring can cause scarring and consequently loss of self-esteem in them and long term sexual dysfunctions due to pain. These affect the quality of reproductive health in affected women and their partners.

From medical point of view, genital human papilloma virus (HPV) infections are alarming because of their association with genital malignancies in women. There are high- risk oncogenic HPV types that are the cause of all, or nearly all invasive cervical cancers, both the squamous cell and adenocarcinoma.High risk HPV types also contribute to vulvar and vaginal cancer.

Symptomatic or asymptomatic genital human papilloma virus (HPV) infection is commonest viral sexually transmitted disease in women, with the highest prevalence following onset of sexual activity. As the lifetime risk for acquiring genital HPV infections is in the order of $80 \%$, it is not possible to define high-risk populations for infection. Prevalence rates in the late teens and early twenties are quoted to be as high as 40\% in literature. According to a study, done in 1997 in the United States, $10 \%$ of the US population had an HPV infection, 4\% had cervical cytology abnormalities and $1 \%$ had visible genital warts at one point-in-time analysis. $^{2}$

Most patients infected with HPV remain asymptomatic as their body's natural immune system controls the infection. The young women with intact immune systems are highly efficient in eliminating these viruses .According to a study, it was seen that $70 \%$ of a population had cleared the HPV after 1 year and more than $90 \%$ after 2 years. ${ }^{3}$ However, in a small minority of population genital human papilloma virus infections persist. In unscreened population, about $2 \%$ patients show the anogenital wart or pre invasive or invasive cervical lesion. If left untreated, the course of genital human papilloma virus infections varies. Genital warts 
may regress spontaneously in $10-30 \%$ of patients and in others, it may remain unchanged or multiply and increase in size. ${ }^{4}$ Genital warts are associated with the problems such as pain, itching, and bleeding and significant psychosocial discomfort. Patients who are immuno-compromised are more likely to develop symptomatic disease and typically have larger warts for a longer duration than immuno-competent patients infected with the virus. ${ }^{5}$

Similarly, when HPV is associated with cellular abnormalities, the majority of infections (80\%) clear spontaneously within 12-18 months. In minority of individuals $(20 \%)$, and over time the HPV genome may integrate itself into host cell DNA. This results in transcription of oncoproteins, cell cycle progression, multiplication of genetic mistakes, genetic instability, and emergence of a malignant phenotype. ${ }^{6}$ This forms the basis of major medical concern, which is the prevention of lower genital tract malignancies. During the productive phase of genital human papilloma virus infections the cytology may show evidence of viral changes or low grade abnormalities .Histological examination may reveal signs of viral infections or cervical intraepithelial neoplasia, grade I (CIN I). Currently, the mode of treatment for women persistently infected with high risk HPV types is colposcopy and biopsy. The conization of cervix is reserved for patients with CIN II or III proven by biopsy. This is highly effective method of treatment that has contributed to the remarkable reduction in the numbers of women with invasive cervical cancer and death from cervical cancer in the United States In a screened population , $<2 \%$ of high-risk HPV infections ultimately lead to cervical carcinoma whereas this figure rises to $5 \%$ in an unscreened patients. ${ }^{7} \mathrm{HPV}$ $16 \& 18$ are responsible for approximately $55 \%$ and $11 \%$ of cervical cancers respectively and have been designated as carcinogenic agents by the International Agency for Research on Cancer ${ }^{8}$

\section{Virology}

HPV is a small stranded intracellular DNA virus. It lacks outer lipid containing viral membrane. It is species - and tissue-specific virus that targets the epithelial cells of the lower genital tract. Till date over $100 \mathrm{HPV}$ types have been identified. The distinction between different HPV types is based upon differences in DNA sequences. ${ }^{9}$ They infect either mucosal or cutaneous surface and cause visible benign lesions referred as genital warts (condyloma acuminata) .Genital warts have the potential to progress into pre-invasive or invasive cancers of lower genital tract depending on the type of HPV involved. Different HPV types fall into two discrete groups according to their oncogenic potential. Those associated with benign wart formation, such as HPV-6 and HPV-11, are known as low risk HPV (10) and the one associated with cellular abnormalities and subsequent neoplasia, such as HPV-16 and HPV-18 as high- risk viruses. ${ }^{11}$ The low risk types HPV, which are associated with the development of external genital warts, are most frequently transmitted via direct skin contact with infected lesions. ${ }^{12}$ The lesions like Bowenoid papulosis, Bowen's disease of the genitalia and cervical cancer are among the diseases associated with high risk HPV types. These are the ones associated with cellular abnormalities. ${ }^{13}$ High risk oncogenic HP types cause flat lesions which are nearly invisible. These are the ones which cause nearly all, almost $99.7 \%$ invasive cancers, both squamous and adenocarcinoma. High -risk HPV types also contribute to vulvar and vaginal cancer. ${ }^{14}$

\section{Immune Responses to HPV Infection}

Natural HPV infection is poorly immunogenic; it is confined to epithelium and induces little inflammation, thereby frequently evading immunological control; but in immuno-compromised individuals HPV disease is seen in high incidence. Neutralizing antibodies to the L1 or L2 capsid proteins are HPV type specific. Natural infection with HPV in the human produces an immune response to the $\mathrm{L} 1$ protein. Once infection occurs, viral proteins synthesized within the host cells are processed into peptides and presented by major histocompatibility complex (MHC) molecules. As they are polymorphic, different individuals will display different peptides from the same proteins. The viral peptides displayed on the cell surface are seen as foreign antigens and are the targets for a cell mediated immune response involving virus specific CD8+ cytotoxic T lymphocytes (CTLs) and CD4+ T helper lymphocytes. Antigens displayed on neoplastic epithelial cells are recognized by activated effectors CTLs .Effectors T cells activation requires antigen presentation by antigen-presenting cells, such as dendritic cells. ${ }^{15}$

\section{Natural History of HPV Infection}

The genome of HPV consists of approximately 8000 base pairs. These base pairs are divided into two categories early (E) and late (L) genes. The L1 and L2 open reading frames (ORFs) of the late genes are responsible for the expression of the L1 and L2 capsid proteins, which together make-up the viral capsid. The capsid coat in exposed human results is an antibody response. The E6 and E7 ORFs are the so called oncogenes which encode for E6 and E7 oncoproteins that interact with host cell tumor suppressor proteins. In contrast, the high risk oncogenic viruses are potentially dangerous as they contain two early (E) genes responsible for vulvar, vaginal and, cervical neoplasm to the human host. E2 ORF encodes for a protein that are important in the control of E6 and 
E7 transcription. The high risk oncogenic viruses are potentially dangerous to the human host, for they contain these two early genes- E6and E7 that is responsible for vulvar, vaginal, or cervical neoplasms. E6 and E7 encode transforming proteins that induce cell proliferation and prevent apoptosis of the infected epithelial cells by binding to the tumor suppressor gene products $\mathrm{p} 53$ and pRB. ${ }^{16,17}$.This results in development of disease in some women over time. Over the short term, within weeks and months, visible lesions appear which is referred as the condylomata. Over the months and years, pre-invasive and invasive cancers of the genital tract are discovered on the lower genital tract in women. ${ }^{18}$ This is the most important sequelae of HPV infection. In more than $90 \%$ cases mucocutaneous external genital warts are caused by low risk HPV types 6 and 11.However, it may also be caused by HPV with intermediate- and high oncogenic risk.

High risk oncogenic types of HPV are those which cause anogenital neoplasia .Neoplasia is detected most often in cervix, however, vulva, vagina and anal region may also be affected .Worldwide, $99.7 \%$ of cervical cancers contain high-risk HPV .HPV 16 and 18 are responsible for approximately $55 \%$ and $11 \%$ of cervical cancers respectively and have been designated as carcinogenic agents by the International Agency for research on Cancer, 1995. ${ }^{8}$ In the natural course of HPV infection, the majority of infections up to $80 \%$ clear spontaneously in $12-18$ months. However, infection with high risk viruses takes slightly longer to clear as compared to the low risk ones. During a productive infection, cytology may show evidence of viral changes or low grade abnormalities. Histological examination may show features of viral infection or cervical intraepithelial neoplasia, grade 1(CIN I). However, in minority of cases $(20 \%)$ the infection persists and over time the HPV genome may integrate itself into host cell $\mathrm{DNA}^{19}$ The break for integration occurs almost inevitably through the E2 ORF. This leads to loss of E2 expression and hence, loss of its normal function that is the control of the expression of the E6 and E7 and consequent transcription of the E6 and E7 oncoproteins. As a result The E6 and E7 oncoproteins are expressed in high amounts in high grade disease. Within the cell, E6 inactivates the p53 tumor suppressor protein and E7 inactivates the $\mathrm{pRb}$ protein. This results in cell cycle progression, multiplication of genetic mistakes, genetic instability and emergence of a malignant phenotype and eventual invasion leading to carcinogenesis. ${ }^{20}$

The cellular abnormalities seen in HPV - infected squamous cells are cytoplasmic vacuolization, nuclear enlargement, irregularity and hypochromasia; whereas, histological examination may reveal signs of viral infection or cervical intra-epithelial neoplasia.

\section{Epidemiology}

Most patients acquire one or more types of HPVs during their lifetime and remain asymptomatic. The peak age of acquiring these infections in women is 20-29 years. ${ }^{16}$ After the age 30 years, the incidence of HPV infections decreases and incidence of cervical cancer increases. ${ }^{21}$ There are various factors which affect the acquisition of HPV virus. In a recent study consistent use of condoms has not been shown to be associated with decreased risk of HPV acquisition, as expected. ${ }^{22}$ However, in another study, the incidence of genital HPV was 37.8 per 100 patient -years at risk in women whose partner used condoms as compared to 89.3 per 100 patient -years at risk in those women whose partners used condoms in less than $5 \%$ of the time. ${ }^{23} \mathrm{Also}$, in women whose partners were consistent with condom use did not show cervical intraepithelial lesions. $^{24}$

Besides, there are number of factors that influence the rate of patient elimination of HPV. Younger women, aged less than 24 eliminate these viruses more efficiently than those more than 30 years. ${ }^{25}$

\section{Treatment Strategies}

The standard treatments for genital warts are topical application of podophyllin paint, podophyllotoxin gel, topical trichloroacetic acid or laser therapy. These agents physically or chemically destroy the exophytic tumor. Another option for destruction of these warts is sublesional or intralesional injection of interferon. ${ }^{26}$ Recently, the use of imiquimod has been reported. Imiquimod is applied locally but, it works by enhancing the patient's local immune system by stimulating the host's production of interferon and tumor necrosis factor. ${ }^{27}$ Besides, recently, HPV vaccines have become available. These provide a prophylaxis for HPV infection. ${ }^{28}$ There are two types of vaccine available; one is a quadrivalent against HPV 6/11/16/18; and other is bivalent against HPV 16/18. ${ }^{29}$

Currently, there is limited public awareness of HPV as a common sexually transmitted infection and its links with cervical intraepithelial lesions and its progression into cervical neoplasia. ${ }^{30}$ Therefore, the public awareness regarding genital human papillomavirus infections in the form of genital wart, its course, spontaneous clearance, regression and development of cervical intraepithelial lesions as the sequelae of high risk HPV infections needs to be enhanced .Also, different modalities of prophylactic measures and treatment methods needs to be offered to these women by the health workers, so as to improve their quality of reproductive health . 


\section{References}

1. Centers for Disease Control and Prevention. Genital HPV infection- CDC fact sheet .2004

2. Koutsky LA. Epidemiology of genital human papillomavirus infection. Am J Med 1997; 102 (Suppl): 3-8.

3. Ho GYF, Bierman R, Beardsby L, et al. Natural history of cervicovaginal human papillomavirus infection in young women. N Engl J Med 1998; 338:423-428.

4. Stone KM . Human papillomavirus infection and genital warts; update on epidemiology and treatment. Clin Infect Dis 1995 ; 20 Suppll1: 91-7.

5. Tindle RW, Frazer IH. Immunology of anogenital human papillomavirus (HPV) infection . Aust NZ J Obstet Gynaecol 1990; 30: 370-5.

6. Peto J, Gilham C, DeaconJ et al. Cervical HPV infection and neoplasia in a large populationbased prospective study; the Manchester cohort. Br J Cancer 2004; 91: 942-53.

7. Einstein $\mathrm{MH}$, Schiller Jt et al . Clinicians guide to human papilloma virus immunology: knowns a\& unknowns . Lancet Inf Dis 2009; 9: 347- 356.

8. Munoz N, Bosch FX, de Sanjose S, et al. Epidemiologic classification of human papilloma virus types associated with cervical cancer. $\mathrm{N}$ Engl J Med 2003;348: 518-527.

9. Howley P M, Schlegel R. 1985 The human papilloma viruses. American Journal of Medicine 1985; 25: 155-158

10. Ferenczy A. Epidemiology and clinical pathophysiology of condylomata acuminate. Am J Obstet Gynaecol 1995; 172:1331-9.

11. Stanley M. The immunology of genital human papillomavirus infection. Eur J Dermatol 1998; 8 Suppl. 7: 8-12.

12. Philips TJ, Dover JS. Recent advances in dermatology. N Engl J Med 1992 Jan 16; 326 :167-78.

13. Majewsky S, Jablonska S. Immunology of HPV infection and HPV-associated tumors. INT J Dermatol 1998; 37: 81-95.

14. Wallboomers JM, Jacobs MV, Manos MM et al. Human papilloma virus is a necessary cause of invasive cervical cancer worldwide. J Pathol 1999; 189:12-19.

15. Kirwan MJK, Herrington CS. Human papilloma virus and cervical cancer: Where are we now ? Br J Pbstet Gynaecol 2001:108: 1202-1213.

16. Syrjanen KJ. Human papillomavirus lesions in association with cervical dysplasias and neoplasias Obstet Gynaecol 1983; 62: 617-24.

17. Mosciki AB, Schiffman M, Kjaer S et al. Chapter 5: Updating the natural history of HPV and anogenital cancer. Vaccine 2006; 24: 542-51.

18. Clifford GM, Gallus S, Herrero R, etal; Worldwide distribution of human papilloma virus types in in cytologically normal women in the International Agency for Research on Cancer HPV prevalence sueveys : a pooled analysis . Lancet 2005; 366: 991-8.

19. Frazer IH, Thomas R, Zhou J et al. Potential strategies utilized by papilloma virus to evade host immunity .Immunol Rev 1999: 168:13142.

20. Greenberg MD, Rutledge LH, Reid R et al. A double - blind randomized trial of $0.5 \%$ podofilox and placebo for the treatment of genital warts in women. Obstet Gynaecol 1991; 77: 735-748.

21. Office of Population Censuses and Surveys 1994 Cancer statistics : registration in England and Wales 1989.MBI no.22.HMSO, London

22. Winer RL, Hughes JP, Lee SK, Hughes JP et al. Genital human papilloma virus : infection , incidence and risk factors in a cohort of female university students. Am J Epidemiol 2003; 157:218-226.

23. Hildesheim A, Schiffman MH, Gravitt PE et al. Persistence of type specific human papilloma virus among cytologically normal women.J Infect Dis 1994; 169: 235-240

24. Winer RL, Hughes JP, Feng Q et al. Condom use and risk of genital human papilloma virus infection in young women. N Engl J Med 2006;354: 2645-2654.

25. Van RM, Kaplan JB, Burk RD et al. Phylogenetic classification of human papilloma viruses : Correlation with clinical manifestations . J Gen Virol $1992 ; 73: 2653-2660$.

26. Eron LT, Judson F, Tuckers S et al. Interferon therapy for condyloma acuminate. N Engl J Med 1986; 315: 1059-1064.

27. Tyring SK, Arany I, Stanley MA et al. A randomized, controlled molecular study of condyloma acuminate clearance during treatment with imiquimod. J Infect Dis 1998; 178: 551555 .

28. Adams M, Borysiewicz L, Fiander A et al . Clinical studies of human papilloma vaccines in pre-invasive and invasive cancer. Vaccine 2001; 19: 2549-56.

29. Taira AV, Neukarmans CP, Sanders GD. Evaluating human papilloma virus vaccination programs . Emerg Infect Dis 2004; 10: 1915-23.

30. Peto J, Gilham C, Fletcher O et al. The cervical cancer epidemic that screening has prevented in the UK. Lancet 2004 ;364:249-256. 